\title{
Robust Classification based on Correlations between Attributes*
}

\author{
Alexandros Nanopoulos ${ }^{1 \dagger}$ \\ Apostolos N. Papadopoulos ${ }^{1}$ \\ Tatjana Welzer-Druzovec ${ }^{2}$ \\ Yannis Manolopoulos ${ }^{1}$ \\ ${ }^{1}$ Aristotle University of Thessaloniki, Greece \\ ${ }^{2}$ University of Maribor, Slovenia
}

\begin{abstract}
The existence of noise in the data significantly impacts the accuracy of classification. In this paper, we are concerned with the development of novel classification algorithms that can efficiently handle noise. To attain this, we recognize an analogy between $k$ nearest neighbors $(k \mathrm{NN})$ classification and user-based collaborative filtering algorithms, as they both find a neighborhood of similar past data and process its contents to make a prediction about new data. The recent development of item-based collaborative filtering algorithms, which are based on similarities between items instead of transactions, addresses the sensitivity of userbased methods against noise in recommender systems. For this reason we focus on the item-based paradigm to provide improved robustness, compared to $k \mathrm{NN}$ algorithms, against noise for the problem of classification. We propose two new item-based algorithms, which are experimentally evaluated with $k \mathrm{NN}$. Our results show that, in terms of precision, the proposed methods outperform $k \mathrm{NN}$ classification by up to $15 \%$, whereas compared to other methods like the C4.5 system, improvement exceeds $30 \%$.
\end{abstract}

\footnotetext{
*Work supported by a bilateral Greek-Slovenian project.

${ }^{\dagger}$ A. Nanopoulos is supported by IKY Post-doctoral scholarship.
} 


\section{INTRODUCTION}

Classification involves the construction of a model, denoted as classifier, for the mapping of data to a set of predefined and non-overlapping classes. The accuracy of a classifier is negatively affected by the existence of noisy data, that is, data records with incorrect attribute values. The reason is that noise confuses the learning process and causes the constructed classifier to overfit the data (Han \& Kamber, 2000). In this paper we are interested in developing novel classification algorithms that are robust and construct classifiers with significantly improved accuracy in the presence of noise.

The development of classification algorithms that handle noise is a worthwhile problem, due to the direct impact that noise has on the reliability of data analysis' results and, thus, on decision making. Controllable data-acquisition procedures can limit the amount of noise, rendering it easily resolvable with preprocessing techniques (Pyle, 1999). In other situations, however, the intrusion of noise cannot be restrained; for instance, data that are collected online in e-commerce sites (e.g., surveys, questionnaires, purchases, etc.). It has been reported (O'Mahony, Hurley, Kushmerick, \& Silvestre, 2004) that to a large degree the visitors of e-commerce sites tend to deliberately provide false values, for reasons varying from privacy concerns to "shilling" attacks (O'Mahony et al., 2004). In such cases preprocessing techniques are not effective. What is needed is classification algorithms that can tackle noise. ${ }^{1}$

Existing classifiers can be divided into two categories (James, 1985), eager and lazy. In contrast to an eager classifier (e.g., decision tree), a lazy classifier (Aha, 1997) builds no general model until a new sample arrives. A $k$-nearest neighbor $(k N N)$ classifier (Dasarathy, 1991) is a typical example of the latter category. It works by searching the training set for the

\footnotetext{
${ }^{1} \mathrm{~A}$ racy account of the people's intention to provide false data when being asked, is described by Witten et al. (Witten, Moffat, \& Bell, 1999): when the authors ask their students to give their birthdate after they have explained to them that they want to demonstrate the "birthday paradox", it takes 366 tries to find two students with the same birthdate!
} 
$k$ nearest neighbors of the new sample and assigns to it the most common class among its $k$ nearest neighbors. In general, a $k \mathrm{NN}$ classifier has satisfactory noise-rejection properties. Other advantages of a $k \mathrm{NN}$ classifier are that it (a) is analytically tractable, (b) for $k=1$ and unlimited samples the error rate is never worse than twice the Bayes' rate, and (c) it is simple to implement.

Due to the aforementioned characteristics, $k \mathrm{NN}$ classifiers are very popular and find many applications. Nevertheless, although they present adequate efficiency for low amount of noise, our results (Section) indicate that their performance degrades for medium and high noise. Interestingly enough, an analogous conclusion has been reported (Sarwar, Karypis, Konstan, \& Riedl, 2001) for the seemingly different problem of user-based (UB) collaborative-filtering (CF). ${ }^{2}$ Albeit the different objectives between a $k \mathrm{NN}$ classifier and a UB CF algorithm, they both have a common way of working: given a new datum (record and user-transactions, respectively), they first find a neighborhood of similar past data and, then, they process its contents to make predictions about the new datum. The bottleneck in this procedure is the search for neighbors among a large population of potential neighbors (Herlocker, Konstan, Borchers, \& Riedl, 1999).

\section{Motivation}

The inefficiency of UB algorithms for significant amounts of noise has recently lead to the development of novel item-based (IB) CF (Sarwar et al., 2001) algorithms which, in contrast to UB ones, base their prediction on similarities between items instead of transactions. UB algorithms are outperformed by IB ones, because the latter can better isolated noise, as in each user-transaction only the items with strong similarities are considered. Therefore, based on the previously described analogy between classification and CF algorithms, the question that motivates our research is: can we involve the paradigm of IB algorithms to build lazy classifiers that are more robust against noise than $k \mathrm{NN}$ classifiers?

\footnotetext{
${ }^{2}$ Note that recommender systems and $\mathrm{CF}$ algorithms, in particular, find many and significant applications (Resnick \& Varian, 1997).
} 


\section{Contribution}

Our contributions are summarized as follows.

- First, we demonstrate that the IB paradigm can be involved to develop robust classifiers.

- We expound that the straightforward adoption of IB methods leads to less accurate classification. Therefore, we develop a novel algorithm, which addresses the deficiencies of straightforward adoption.

- We recognize a trade-off between the robustness of the new IB algorithm against noise and the high precision of $k \mathrm{NN}$ classification in the absence of noise.

- We develop a hybrid algorithm that combines the advantages of both approaches. Our experimental results illustrate that: (i) in the presence of noise the proposed IB algorithm improves the precision of $k \mathrm{NN}$ classification up to $15 \%$; (ii) the hybrid algorithm is a good amalgamation, because it attains precision analogous to a $k \mathrm{NN}$ classifier, when no or low amount of noise is added, and analogous to an IB algorithm, when medium or high amount of noise is added.

The rest of this paper is organized as follows. Section describes related work. In Section we develop the proposed method, whereas in Section we present the experimental results. We conclude this paper in Section .

\section{RELATED WORK}

Due to its simplicity and good performance, $k \mathrm{NN}$ classification has been studied thoroughly (Dasarathy, 1991). Several variations were developed (Atkeson, Moore, \& Schaal, 1997), like the distanceweighted $k \mathrm{NN}$, which puts emphasis on nearer neighbors, and the locally-weighted averaging, which uses kernel width to controls the size of neighborhood that has large effect. Also, $k \mathrm{NN}$ classification has been combined with other methods and, instead of predicting a class with 
simple voting, prediction is done by another machine learner (e.g., neural-network) (Atkeson \& Schaal, 1995). In our research we are interested in examining the essential characteristics of $k \mathrm{NN}$ and item-based classification, that is, to compare classification based on similarities between samples $(k \mathrm{NN})$ in contrast to similarities between attribute values (item-based). For this reason, to make comparison clearer, we did not examine techniques like the aforementioned ones. Moreover, such techniques (e.g., combination with other machine learners) can be applied for both types of classifiers.

Another category of research that is related to ours is the integration of association-rules mining with classification (Liu, Hsu, \& Ma, 1998). Integration is done by focusing on mining class association rules (CARs), i.e., association rules between items and classes. CARs are used to build an eager classifier, which was experimentally shown to achieve better precision compared to the C4.5 classification system. Basically, CARs correspond to multi-order dependencies between items and class labels. Since the number of CARs may increase rapidly and impact the accuracy of classification, their number is controlled with parameters for minimum support and confidence, and with heuristics for grouping rules. In contrast, we focus on up to secondorder dependencies to develop item-based classifiers. Second-order dependencies are effective in capturing correlations between attributes and classes. Additionally, they make our approach much more simple, because it does not need the tuning of parameters like minimum support and confidence, which, in general, is a difficult task (Han \& Kamber, 2000). The performance of CARs has not been examined with respect to noise. It is interesting to examine, in our future work, if multi-order dependencies help in the presence of noise. Other uses of association rules include the mining of partial classifications (Ali, Manganaris, \& Srikant, 1997). Partial classification does not cover all classes and all examples of any given class, and is used when complete classification is infeasible or undesirable.

In the recent years the field of $\mathrm{CF}$ has found many important applications in real-world cases (Resnick \& Varian, 1997). Although UB CF algorithms are simple and quite effective, 
IB methods (Sarwar et al., 2001) have been proposed to address their limitations, for instance, sensitivity to noise. Nevertheless, to our knowledge, no work has studied the involvement of the IB paradigm to the problem of classification.

\section{DEVELOPING ITEM-BASED CLASSIFIERS}

We first summarize the IB paradigm and describe a simple classifier that is based on its direct implementation. Next, we elaborate further and propose extensions for more effective classification.

\section{Direct implementation of the IB paradigm}

Let $I$ be a domain of items, $D$ the collection of past transactions, and $T=\left\{i_{1}, \ldots, i_{m}\right\}$ the transaction of the target user. An IB collaborative-filtering algorithm finds for each item $i_{j} \in T$ $(1 \leq j \leq m)$ the set $N\left(i_{j}\right)$ of $k$ items with which $i_{j}$ is most correlated within transactions of $D$. The items which belong to $\bigcup_{j=1}^{m} N\left(i_{j}\right)-T$ (i.e., excluding those contained in $T$ ) are recommended to the user. Correlation between two items $i_{1}$ and $i_{2}$ can be measured in several ways (Han \& Kamber, 2000): (a) for items with numerical values the adjusted cosine similarity measure has been proposed, (b) for nominal items a commonly used measure is confidence. The confidence is an estimator for the probability $P\left(i_{2} \mid i_{1}\right)$ and is computed as: $\operatorname{conf}\left(i_{1}, i_{2}\right)=$ $\frac{\operatorname{supp}\left(i_{1}, i_{2}\right)}{\operatorname{supp}\left(i_{1}\right)}$, where $\operatorname{supp}\left(i_{1}, i_{2}\right)$ and $\operatorname{supp}\left(i_{1}\right)$ denote the support of itemsets $\left\{i_{1}, i_{2}\right\}$ and $\left\{i_{1}\right\}$, respectively.

A classifier that directly follows the IB paradigm has to take into account the correlations between attribute values in the training samples. Each such sample $S=\left\{v_{1}, \ldots, v_{m}, c\right\}$, where $v_{i}$ is the value of $i$-th attribute and $c$ is the class label, can be considered as a multidimensional transaction. For simplicity we henceforth assume that the attributes are either nominal or have been discretized. ${ }^{3}$ Using the confidence measure, we find, for each attribute value $v_{i}$, the set

\footnotetext{
${ }^{3}$ Extension to continuous attributes is possible with works (Srikant \& Agrawal, 1996) that find correlations
} 
$N\left(v_{i}\right)$ of the $k$ attribute values that are most correlated with $v_{i}$ in the training samples (we allow the inclusion of $v_{i}$ itself in $\left.N\left(v_{i}\right)\right)$. This way, for a new sample $X=\left\{v_{1}, \ldots, v_{m}\right\}$, we form the set $U(X)=\bigcup_{v_{i} \in X} N\left(v_{i}\right)$. To predict the class of $X$, we also have to find for each $v_{j} \in U(X)$ the set $N^{\prime}\left(v_{j}\right)$ of the $k^{\prime}$ class labels that are most correlated with $v_{j}$. (In our implementation we set $k^{\prime}=1$.) Correlation in this case reflects $P\left(c \mid v_{j}\right)$ and is measured as: $\operatorname{conf}\left(v_{j}, c\right)=\frac{\operatorname{supp}\left(v_{j}, c\right)}{\operatorname{supp}\left(v_{j}\right)}$. For each class label $c$, we compute sum the $\sum_{v_{j} \in U(X)} \operatorname{conf}\left(v_{j}, c\right)$. Finally, we select for prediction the class with the greatest sum.

The direct algorithm is denoted as Item-based classifier (IBC). To understand the intuition behind IBC we consider that: even when several attributes values in the training set have noise, IBC focuses only on the attributes values that (a) are correlated with those in the new sample, and (b) can confidently indicate a class. Thus, IBC more effectively avoids noisy attributes values, because they tend to form weaker correlations and are less frequently included in the $U(X)$ set. In contrast, when $k \mathrm{NN}$ algorithm finds the nearest neighbors, it takes into account the noise in the attribute values while computing the similarities. Nevertheless, IBC has two problems:

1. It is based on first-order correlations between attribute values and class labels. This is restrictive, since higher-order dependencies may improve the accuracy of classification.

2. $U(X)$ has the propensity of getting large. This may be desirable for IB CF algorithms (for higher recall). However, it can hinder the accuracy of IBC, especially when combined with the 1st problem, because the more focused $U(X)$ is, the better the prediction becomes.

In the following of this section we develop solutions to the aforementioned problems. Regarding the execution time, IBC needs to maintain the support values that are required to compute the confidence values when a new sample arrives. This can be done easily with two-dimensional arrays or with trie structures (Han \& Kamber, 2000), which can be easily updated with insertions/deletions of samples. Therefore, IBC classifies a new sample in lazy manner and the between quantitative items. 
pre-computed support values play a role analogous to that of an index structure that speeds-up the finding of nearest neighbors (Papadopoulos \& Manolopoulos, 2005) in $k$ NN classifiers.

\section{Pairwise-based classifier}

Initially we consider the first problem of IBC. As described, CAR classifiers (Liu et al., 1998) detect dependencies of various orders between the attribute values and the classes labels, but require careful tuning of several parameters. As a conciliation between first-order dependencies in IBC and multi-order dependencies in CAR-like methods, we choose to consider up to secondorder dependencies, which: (a) can effectively capture many associations between attribute variables and class labels; (b) are simple and do not require parameter tuning; and (c) do not present significant storage overhead, in contrast to storing much more many support values that would be required to calculate multi-order dependencies. Because we want to estimate probabilities of the form $P\left(c \mid\left\{v_{i}, v_{j}\right\}\right)\left(\left\{v_{i}, v_{j}\right\}\right.$ corresponds to a 2-itemset) through $\operatorname{conf}\left(\left\{v_{i}, v_{j}\right\}, c\right)$ values, we find and store supports of the form $\operatorname{supp}\left(\left\{v_{i}, v_{j}, c\right\}\right)$ from the training samples.

With respect to the second problem of IBC, given a new sample $X=\left\{v_{1}, \ldots, v_{m}\right\}$, we propose the following method for the formation of $U(X)$. For each $v_{i} \in X$, we include in $U(X)$ only those $v_{j} \in N\left(v_{i}\right)$ for which it also holds that $v_{j} \in X$, that is, we concentrate on pairs from the new sample $X$. The latter condition restricts the size of $U(X)$ compared to the case of IBC. Differently from IBC, we keep for each $v_{j} \in U(X)$ the associated $v_{i}$. This way, $U(X)$ contains pairs of the form $\left(v_{i}, v_{j}\right)$, that are tested against each class label $c$ by computing $\operatorname{conf}\left(\left\{v_{i}, v_{j}\right\}, c\right)$ as described previously. ${ }^{4}$ The confidence measures for each class are summed and the class with the greatest sum is selected for prediction. The resulting algorithm is denoted as Pairwise-based classifier (PBC).

The incentive to the design of the PBC algorithm is described as follows. We want to base our prediction on second-order dependencies between attribute values and class labels.

\footnotetext{
${ }^{4}$ Similarly to IBC, we allow $v_{i}$ to be included in $N\left(v_{i}\right)$ as $\left(v_{i}, v_{i}\right)$. When it is tested against a class $c$, we compute $\operatorname{conf}\left(v_{i}, c\right)$, which corresponds to first-order dependency.
} 
Therefore, within a record we have to consider the confidence measure between each pair of attribute values and all class labels. To avoid the impact of noise, we take into account only the pairs of attribute values for which there is a strong correlation between them. To achieve this, for each attribute value in a record, we select between the remaining ones only those that belong to its neighborhood. This way, as described, we manage to both consider second-order dependencies and to restrain the length of $U(X)$, by considering pairs with strongly correlated items.

An example of PBC is depicted in Figure 1. The data table contains five training samples over three attributes $(A, B, C)$ and one class label. The new sample is $X=\left\{a_{1}, b_{2}, c_{2}\right\}$, depicted in the last row. Assuming that $k=2$, the neighborhood sets for each attribute values of $X$ are depicted in the upper-right part of the figure. For instance, $N\left(a_{1}\right)=\left\{a_{1}, b_{1}\right\}$, because $\operatorname{conf}\left(a_{1}, a_{1}\right)=1$ and $\operatorname{conf}\left(a_{1}, b_{1}\right)=3 / 4$ are the highest two for $a_{1}$. The resulting $U(X)$ set is depicted bellow. For instance, associated with $a_{1}$ in $U(X)$ is only $a_{1}$ itself (forming the pair $\left.\left(a_{1}, a_{1}\right)\right)$. Although $b_{1} \in N\left(a_{1}\right)$, it does not belong in $X$ and is excluded from $U(X)$. The same applies for $c_{1}$ with respect to $N\left(b_{2}\right)$, whereas both $c_{2}$ and $a_{1}$, which belong in $N\left(c_{2}\right)$, are included in $U(X)$ associated with $c_{2}$. For each member of $U(X)$, PBC examines the confidence values against the two class labels $l_{1}$ and $l_{2}$. For instance, for $\left(a_{1}, a_{1}\right)$ we compute a $\operatorname{conf}\left(a_{1}, l_{1}\right)=$ $\frac{\operatorname{supp}\left(\left\{a_{1}, l_{1}\right\}\right)}{\operatorname{supp}\left(a_{1}\right)}=3 / 4$, which checks a first-order dependency between $a_{1}$ and $l_{1}$. In contrast, for pair $\left(c_{2}, a_{1}\right)$ we check a second-order dependency by computing $\operatorname{conf}\left(\left\{c_{2}, a_{1}\right\}, l_{1}\right)=\frac{\operatorname{supp}\left(\left\{\left\{a_{1}, c_{2}, l_{1}\right\}\right)\right.}{\operatorname{supp}\left(\left\{a_{1}, c_{2}\right\}\right)}=$ $2 / 2=1$. Taking the sum of confidence values for each class, we get $\operatorname{Sum}\left(l_{1}\right)=2.75$ and $\operatorname{Sum}\left(l_{2}\right)=1.25$, thus we assign the new sample to class $l_{1}$.

\section{Extensions of the pairwise-based classifier}

The assigning of class labels based on the greatest sum value, a method that is denoted as majority voting, can impact the accuracy of $\mathrm{PBC}$ in the presence of noise, when some classes have much more samples in the training set than the others (skewed class distribution). Since 


\begin{tabular}{|c|c|c|c|c|}
\hline & $\mathbf{A}$ & B & C & $\begin{array}{l}\text { Class } \\
\text { label }\end{array}$ \\
\hline \multirow{5}{*}{$\begin{array}{c}\text { train } \\
\text { samples }\end{array}$} & $a_{1}$ & $\mathrm{~b}_{1}$ & $\mathrm{c}_{2}$ & $l_{1}$ \\
\hline & $a_{1}$ & $b_{1}$ & $\mathrm{c}_{1}$ & $l_{1}$ \\
\hline & $a_{1}$ & $b_{1}$ & $\mathrm{c}_{2}$ & $1_{1}$ \\
\hline & $\mathrm{a}_{1}$ & $\mathrm{~b}_{2}$ & $\mathrm{c}_{1}$ & $\mathrm{l}_{2}$ \\
\hline & $\mathrm{a}_{2}$ & $\mathrm{~b}_{2}$ & $\mathrm{c}_{1}$ & $l_{2}$ \\
\hline new sample & $a_{1}$ & $b_{2}$ & $c_{2}$ & ? \\
\hline
\end{tabular}

$\mathrm{N}\left(\mathrm{a}_{1}\right)=\left\{\mathrm{a}_{1}, \mathrm{~b}_{1}\right\}, \quad \mathrm{N}\left(\mathrm{b}_{2}\right)=\left\{\mathrm{b}_{2}, \mathrm{c}_{1}\right\}, \quad \mathrm{N}\left(\mathrm{c}_{2}\right)=\left\{\mathrm{c}_{2}, \mathrm{a}_{1}\right\}$

$U(X)=\left\{\left(a_{1}, a_{1}\right),\left(b_{2}, b_{2}\right),\left(c_{2}, c_{2}\right),\left(c_{2}, a_{1}\right)\right\}$

$\operatorname{conf}\left(a_{1}, l_{1}\right)=3 / 4$

$\operatorname{conf}\left(\mathrm{b}_{2}, \mathrm{l}_{1}\right)=0$

$\operatorname{conf}\left(\mathrm{c}_{2}, \mathrm{l}_{1}\right)=1$

$\operatorname{conf}\left(\left\{c_{2}, a_{1}\right\}, I_{1}\right)=1$

$\operatorname{Sum}\left(I_{1}\right)=2.75$

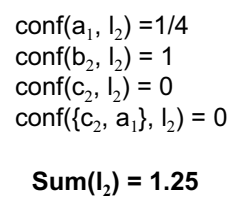

Figure 1: Example of PBC.

larger classes get a larger sum value more easily, PBC tends to bias its prediction towards them, thus the accuracy for smaller classes reduces. To overcome this problem we use a simple variation of the majority voting: Instead of selecting the class with the greater sum of confidence values, we select the one with the largest deviation from the expected value of this sum. In particular, for each class $c$, we take into account two sums, $S_{1}(c)$ and $S_{2}(c) . S_{1}(c)$ is computed as described previously. For $S_{2}(c)$, each time a confidence for class $c$ is computed, we add to $S_{2}(c)$ the value $\frac{1}{\operatorname{supp}(c)}$. In the example of Figure 1 , when we compute conf $\left(a_{1}, l_{1}\right)$, we add to $S_{1}$ the value $3 / 4$, and to $S_{2}$ the value $1 / \operatorname{supp}\left(l_{1}\right)=1 / 3$. In the end, we select the class with the largest difference $S_{1}(c)-\alpha S_{2}(c)$. The $\alpha$ parameter helps to to control the bias: e.g., with $\alpha=0$ (or even $\alpha<0$ ) the larger classes are favored, whereas with $\alpha>0$ the smaller classes are favored. Its tuning will be explained in Section .

The characteristics of $\mathrm{PBC}$ make it more robust against noise, compared to IBC and $k \mathrm{NN}$ classifiers. Nevertheless, by careful experimentation we have recognized a tradeoff between the good performance of $k \mathrm{NN}$ classifiers when no additional noise is added and the robustness of PBC when the amount of noise is significant. For this reason we propose a hybrid approach. When a new sample $X$ arrives, we first apply $k \mathrm{NN}$ classification. If all $k$ nearest neighbors belong to the same class, then we assign $X$ to this class. Otherwise, at the moment that a neighbor with different class has been found, we stop the $k \mathrm{NN}$ classifier and apply PBC. The resulting algorithm is denoted as Hybrid-based classifier (HBC). The intuition behind HBC is that when $k \mathrm{NN}$ reaches a unanimous decision, then low or no noise exists (noise tends to limit 
such unanimous decisions). For this reason, the prediction of $k \mathrm{NN}$ classifier is preferred in these cases. Otherwise, PBC is preferred, because it offers better robustness against noise. This way, HBC combines the advantage of both approaches.

\section{PERFORMANCE RESULTS}

We examined experimentally the performance of the described classifiers: $k \mathrm{NN}, \mathrm{IBC}, \mathrm{RBC}$, and HBC, which we implemented in $\mathrm{C}++$. For comparison purposes, we also examined classification with C4.5 decision-tree, which is denoted as DT. We selected the precision of prediction as our performance measure. For $k \mathrm{NN}$ classification we used the Jaccard similarity measure (nominal attributes). To tune $\alpha$ for $\mathrm{RBC}$ and $\mathrm{HBC}$, we set it with values between -1 and 0 for low noise values (less than 20\%; see below). For higher noise values, we set it to 1 to reduce bias against smaller classes, as described in Section . We tested several values for the $k$ parameter (for all methods besides DT). When noise is added, in some cases precision increases with larger $k$ values, whereas in some others it decreases. For this reason in each measurement we selected the best results for $k$ between 3 and 10 (larger values leaded to non stable precision).

We used the following real data sets from the UCI Machine Learning Repository ${ }^{5}$ : Congressional Voting Records Database (denoted as Voting), Mushrooms Database (denoted as Mushrooms), Nursery Database (denoted as Nursery), and Pittsburgh Bridges Database (denoted as Bridges). All the data sets contain nominal attributes. The smallest, in number of rows, set (Bridges) contains 108 samples, whereas the the largest (Nursery) 12,960. The distribution of classes was more skewed in the Bridges data set than in the others.

Noise is added in two ways: (1) We modify each attribute value (not the class labels) with probability denoted as noise, which is a parameter. We focused on random noise and when a value is replaced, we select one of the remaining ones with uniform probability. (2) We modify the class labels (not the attribute values) with probability equal to noise. Again, when we

\footnotetext{
${ }^{5}$ http://www.ics.uci.edu/ mlearn/MLRepository.html
} 
replace a class label, we select one of the others with uniform probability. The two types of noise, which are denoted as attribute noise and class noise, respectively, are different and we want to examine both of them.

First, we tested the Voting data set against attribute noise. We measured precision with 10-cross validation. The results with respect to the noise parameter are given in Figure 2a. IBC, due to the two problems that are described in Section, has the lower precision. As expected, DT performs well when noise $=0$. However, its precision is reduced significantly as noise increases. The comparison between $k \mathrm{NN}$ and $\mathrm{PBC}$ illustrates the tradeoff that we described in the previous section: for low noise values $k \mathrm{NN}$ performs better. But as noise increases, $\mathrm{RBC}$ due to its robustness becomes better; their difference in precision reaches $9 \%$ for large noise values. HBC tracks each time the best of the latter two algorithms. When noise is low, the precision of $\mathrm{HBC}$ is analogous to that of $k \mathrm{NN}$. As noise increases, HBC behaves similarly to RBC. This explains the motivation for the development of the hybrid approach. For larger values of noise, the precision of all methods besides $k \mathrm{NN}$ converges to the same point, whereas $k \mathrm{NN}$ presents the worst precision for large noise values.

Next, we examined the Mushrooms data set, which contains classes that are, in general, easily detectable. We added attribute noise. To make the comparison more challenging, we selected as evaluation set a $10 \%$ of samples whose majority belongs to the less frequent between the two classes (non-edible) and we used the other $90 \%$ for training. The results are depicted in Figure 2b. For clarity, we omit the results for DT and IBC, because they consistently present the worst performance, compared to the other methods, when noise is added. Similarly to the previous experiment, the same tradeoff is observed. For lower noise values $k \mathrm{NN}$ outperforms $\mathrm{RBC}$, whereas the latter becomes better when noise increases. HBC again tracks the best of the two algorithms. For low noise it outperforms RBC by $5.5 \%$ and for larger noise its difference from $k \mathrm{NN}$ reaches $12 \%$ (in the same case, the difference between $\mathrm{RBC}$ and $\mathrm{UB}$ is more than $14 \%)$. 


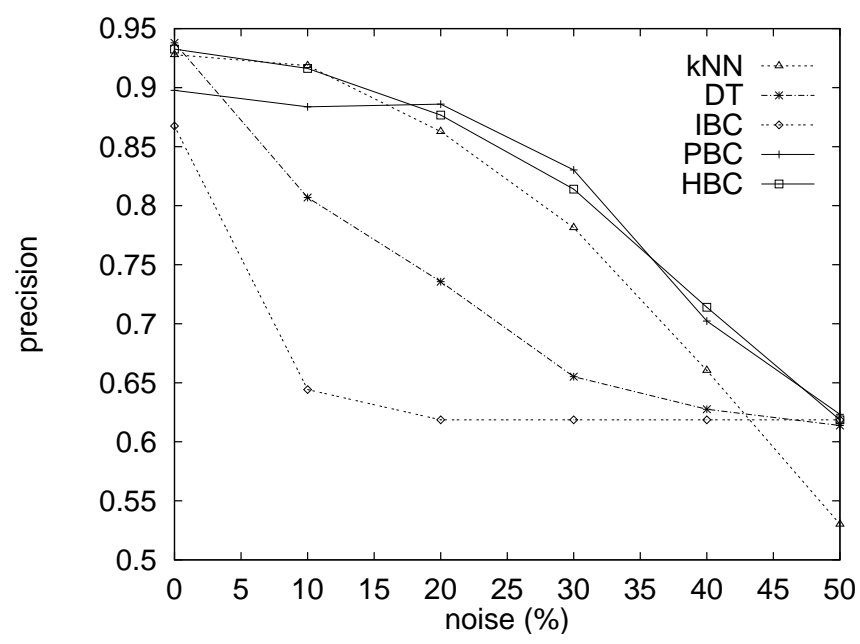

(a)

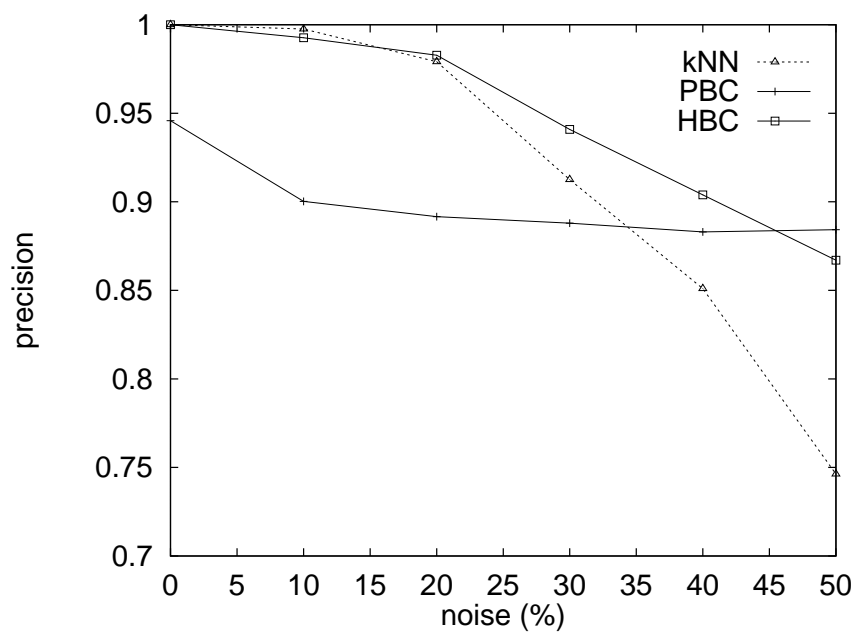

(b)

Figure 2: Precision w.r.t. noise for: (a) Voting, (b) Mushrooms data set. 
Also, we examined the Nursery data set for class noise. The results (using 10-cross validation) are illustrated in Figure 3a, which are similar to the previous ones in terms of relative performance.

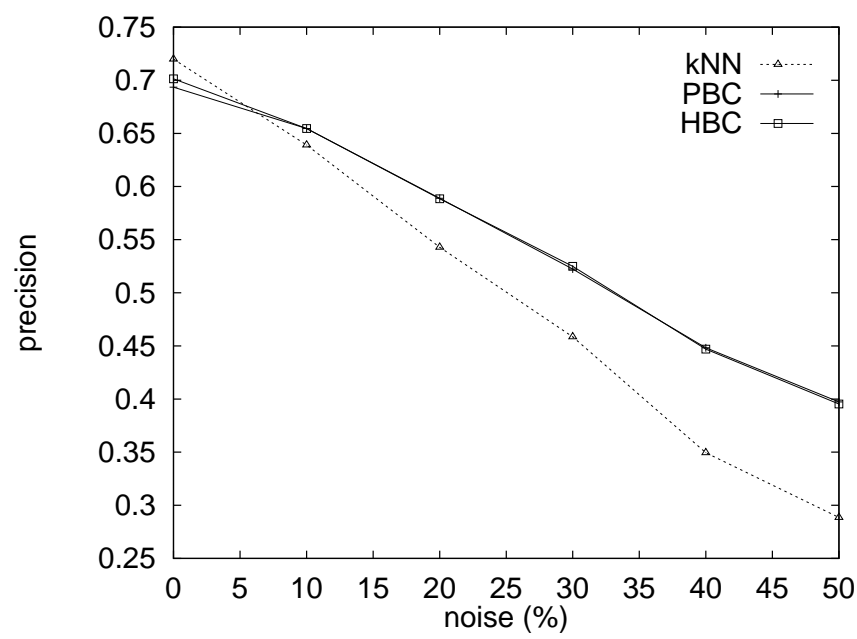

(a)

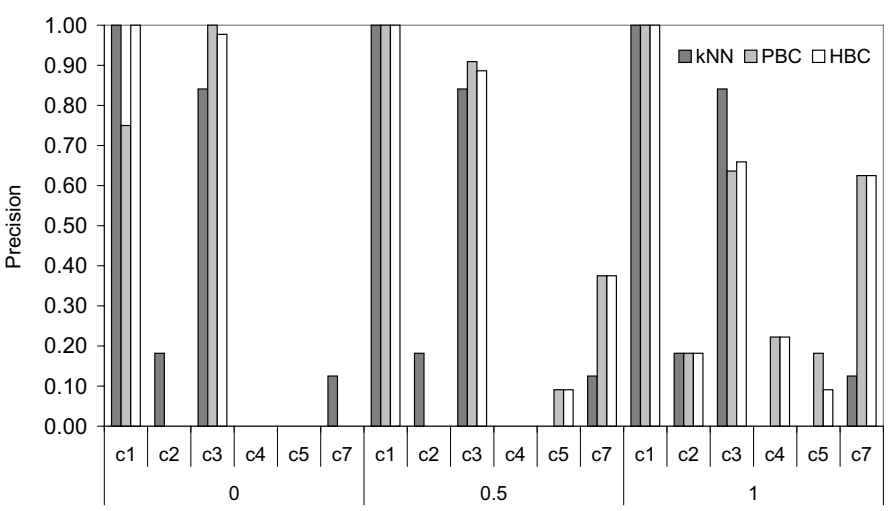

(b)

Figure 3: (a) Precision w.r.t. noise for Nursery data set. (b) Precision for each class in the Bridges data set w.r.t $\alpha$.

Finally, we tested the impact of $\alpha$ for skewed class distribution. As mentioned, when $\alpha=0$, prediction is biased towards the largest classes. As $\alpha$ increases, the bias against less frequent classes reduces. The Bridges data set contains 7 class labels, but we omit the sixth label (it was 'NIL' and was contained by only one sample). Compared to the number of samples of the smallest class $\left(c_{7}\right)$, the largest class $\left(c_{3}\right)$ contains 5 times more samples. The second largest 
$\left(c_{1}\right)$ contains twice more samples. We added no noise in order not to change the existing skew in class distribution. The precision with respect to $\alpha$ is depicted, separately for each class, in Figure 3b. When $\alpha=0$, the largest classes $\left(c_{1}\right.$ and $\left.c_{3}\right)$ are predicted with much larger precision than the others. The $k$ NN classifier achieves a small, but larger non-negligible precision for two of the smaller classes $\left(c_{2}\right.$ and $\left.c_{7}\right)$. For the remaining classes $\left(c_{4}\right.$ and $\left.c_{5}\right)$, all methods result to zero precision. As $\alpha$ increases, $\mathrm{PBC}$ and $\mathrm{HBC}$ are not less biased towards the large classes. For $\alpha=1$, the precision of $\mathrm{PBC}$ and $\mathrm{HBC}$ for $c_{2}$ is analogous to that of $k \mathrm{NN}$, whereas they outperform $k \mathrm{NN}$ for the smallest class $c_{7}$. Moreover, PBC and HBC have a small, but nonnegligible precision for classes $c_{4}$ and $c_{5}$, for which $k \mathrm{NN}$ presents zero precision. On the other hand, the precision of $\mathrm{PBC}$ and $\mathrm{HBC}$ for the largest class $c_{3}$ reduces when $\alpha=1$. Thus, the reduction of bias results to a trade-off between the precision for smaller and larger classes.

\section{CONCLUSIONS}

We propose new algorithms for lazy classification, which follow the item-based paradigm. In contrast to $k \mathrm{NN}$ classification that predicts according to similarities between samples in the training set, item-based classification predicts according to similarities between attribute values. This characteristic makes item-based classification more robust against noise.

Our first algorithm, PBC, resolves the problems of the straightforward adoption of the IB paradigm. Our second algorithm, HBC, is a hybrid approach that combines the advantages of $k \mathrm{NN}$ and $\mathrm{PBC}$ algorithms. Our experimental results show that, in the presence of attribute or class noise, the proposed algorithms outperform $k \mathrm{NN}$ classification in terms of precision by up to $15 \%$. The improvement against the $\mathrm{C} 4.5$ classification system is more than $30 \%$.

In our future work we will extend item-based classification algorithms to consider distance weighting (between attribute values), we will examine mixtures of nominal and continuous attributes, and complex voting methods (e.g., integration with neural networks). 


\section{References}

Aha, D. W. (1997). Editorial. Artificial Intelligence Review (Special Issue on Lazy Learning), $11(1-5), 1-6$.

Ali, K., Manganaris, S., \& Srikant, R. (1997). Partial classification using association rules. In Proc. knowledge discovery and data mining conf. (p. 115-118).

Atkeson, C., Moore, A., \& Schaal, S. (1997). Locally weighted learning. Artificial Intelligence Review, 11(1-5), 11-73.

Atkeson, C., \& Schaal, S. (1995). Memory-based neural networks for robot learning. Neurocomputing, 9, 243-269.

Dasarathy, B. (1991). Nearest neighbor norms: Nn pattern classification techniques. IEEE Computer Society Press.

Han, J., \& Kamber, M. (2000). Data mining: Concepts and techniques. Morgan Kaufmann.

Herlocker, J., Konstan, J., Borchers, A., \& Riedl, J. (1999). An algorithmic framework for performing collaborative filtering. In Proc. acm conf. on research and development in information retrieval (sigir) (p. 230-237).

James, M. (1985). Classification algorithms. John Wiley \& Sons.

Liu, B., Hsu, W., \& Ma, Y. (1998). Integrating classification and association rule mining. In Proc. knowledge discovery and data mining conf. (p. 80-86).

O’Mahony, M., Hurley, N., Kushmerick, N., \& Silvestre, G. (2004). Collaborative recommendation: A robustness analysis. ACM Transactions on Internet Technology, 4(4), 334-377.

Papadopoulos, A., \& Manolopoulos, Y. (2005). Nearest neighbor search: A database perspective. Springer (Series in Computer Science).

Pyle, D. (1999). Data preparation for data mining. Morgan Kaufmann.

Resnick, P., \& Varian, H. R. (1997). Recommender systems (special issue). Communications of the $A C M, 40(3)$.

Sarwar, B., Karypis, G., Konstan, J., \& Riedl, J. (2001). Item-based collaborative filtering 
recommendation algorithms. In Proc. world wide web conf. (p. 285-295).

Srikant, R., \& Agrawal, R. (1996). Mining quantitative association rules in large relational tables. In Proc. acm conf. on management of data (p. 1-12).

Witten, I., Moffat, A., \& Bell, T. (1999). Managing gigabytes: Compressing and indexing documents and images (2nd edition). Morgan Kaufmann. 